\title{
A comparative study of truly incompressible and weakly compressible SPH methods for free surface incompressible flows
}

\author{
Z. Chen ${ }^{1,2}$, Z. Zong ${ }^{1,2}$, M. B. $\operatorname{Liu}^{3, *, \dagger}$ and H. T. $\mathrm{Li}^{1,2}$ \\ ${ }^{1}$ State Key Laboratory of Structure Analysis for Industrial Equipment, Dalian, 116024, China \\ ${ }^{2}$ School of Naval Architecture, Dalian University of Technology, Dalian, 116024, China \\ ${ }^{3}$ Institute of Mechanics, Chinese Academy of Sciences, Beijing 100190, China
}

\begin{abstract}
SUMMARY
In this paper, the performance of the incompressible SPH (ISPH) method and an improved weakly compressible SPH (IWCSPH) method for free surface incompressible flows are compared and analyzed. In both methods, the Navier-Stokes equations are solved, and no artificial viscosity is used. The ISPH algorithm in this paper is based on the classical SPH projection method with common treatments on solid boundaries and free surfaces. The IWCSPH model includes some advanced corrective algorithms in density approximation and solid boundary treatment (SBT). In density approximation, the moving least squares (MLS) approach is applied to re-initialize density every several steps to obtain smoother and more stable pressure fields. An improved coupled dynamic SBT algorithm is implemented to obtain stable pressure values near solid wall areas and, thus, to minimize possible numerical oscillations brought in by the solid boundaries. Three representative numerical examples, including a benchmark test for hydrostatic pressure, a dam breaking problem and a liquid sloshing problem, are comparatively analyzed with ISPH and IWCSPH. It is demonstrated that the present IWCSPH is more attractive than ISPH in modeling free surface incompressible flows as it is more accurate and more stable with comparable or even less computational efforts. Copyright $\odot 2013$ John Wiley \& Sons, Ltd.
\end{abstract}

KEY WORDS: incompressible SPH; weakly compressible SPH; free surface flows; fluid-structure interaction

\section{INTRODUCTION}

SPH is a mesh-free, Lagrangian particle method [1]. It was originally invented to solve astrophysical problems in three-dimensional open space [2] and has drawn more and more attractions in recent years because of its great adaptabilities in modeling solid and fluid dynamic problems [3,4]. In SPH, the physical objects are represented by a set of particles with properties such as masses, velocities, pressures and so on. The particles interact with neighboring ones within a support domain defined by the kernel function. SPH particles can move freely, and there are no restrictions originated from using a grid, and therefore, it is natural in modeling problems with large deformations. It is also convenient to treat free surfaces and moving interfaces. These nice features make SPH method more advantageous over conventional grid-based methods in simulating the fluid flows with changing and breakup of free surfaces, and violent fluid-solid interaction with liquid impact, sloshing and splashing $[5,6]$.

In modeling incompressible flows with free surfaces, the traditional SPH method is based on the assumption that the modeling fluid can be regarded as weakly compressible, and this approach is

\footnotetext{
*Correspondence to: M. B. Liu, Key Laboratory for Mechanics in Fluid Solid Coupling Systems, Institute of Mechanics, Chinese Academy of Sciences, Beijing 100190, China.

†E-mail: liumoubin@imech.ac.cn 
usually referred to as weakly compressible SPH (WCSPH). WCSPH is able to obtain reasonable flow patterns, but it encounters a number of difficulties especially pressure oscillations. However, during recent years, great progresses have been made in WCSPH methods, and the previous defections such as pressure oscillations have been resolved quite well either by using high order SPH approximation schemes such as the corrective smoothed particle method by Chen et al. [7] and the finite particle method by Liu et al. [8,9] or by using a well-tuned artificial viscosity [10]. Density re-initialization $[11,12]$ is another effective approach to reduce large oscillation of the particle pressure and can be applied every several time steps without too much computational cost. Furthermore, the improvements in treating solid boundaries [13] and free surfaces [14] also contribute to WCSPH in better modeling free surface incompressible flows.

Another approach in resolving pressure is to model the free surface incompressible flows as rigorously incompressible rather than weakly compressible as in WCSPH [15]. This leads to the projection-based incompressible SPH (ISPH) method [16, 17]. Unlike WCSPH, the particle density in ISPH remains unchanged, ensuring the incompressibility of the modeling fluid. The pressure is implicitly obtained from solving the pressure Poisson's equation (PPE) rather than from an artificial equation of state as in WCSPH. The PPE is only related on the relative positions and relative velocities between particles without artificial parameters. Meanwhile, the solution of PPE in ISPH is based on the entire computational domain. Therefore, compared with traditional WCSPH methods, the ISPH methods are usually able to obtain much smoother pressure fields [18].

There are several papers offering comparisons between ISPH and WCSPH. These papers compared the traditional WCSPH method and ISPH method and concluded that the ISPH method has overall advantages over WCSPH method [19]. However, these works did not consider the recent improvements in the SPH method especially in correction of particle approximation and enhancements in treatments of solid boundaries and free surfaces, and therefore, the obtained conclusions are insufficient. Mostafa Safdari Shadloo et al. [20] considered a robust WCSPH model and offered its comparison with the ISPH method, but the present numerical examples are not associated with changing and breakup of free surfaces, and violent fluid-solid interactions with liquid impact, sloshing and splashing. These are usually the most important features for violent free surface flows with moving objects.

As the existing results and conclusions from ISPH and WCSPH are usually inconsistent and even controversial, the purpose of this paper is to examine the performance of the classical ISPH method with an improved WCSPH (IWCSPH) method in modeling free surface incompressible flows with diversified numerical examples to make more reliable conclusions. To make ISPH and IWCSPH comparable, in both models, high order SPH approximation schemes and artificial viscosity are not used. The differences of IWCSPH from conventional WCSPH include a simple MSL approach to re-initialize density and an enhanced solid boundary treatment (SBT) algorithm.

The paper is organized as follows. The basic SPH methodology and the governing equations are firstly introduced. The ISPH algorithm, which is based on classical SPH projection method and some common treatments on solid boundaries and free surfaces, is then presented. The following section gives detailed description of an IWCSPH model. This IWCSPH model combines a moving least squares (MLS) algorithm for re-initializing density, some modifications in free surface condition and an improved coupled dynamic SBT algorithm. Three typical numerical examples, including a benchmark test for hydrostatic pressure, a dam breaking problem and a liquid sloshing problem, are comparatively analyzed with ISPH and IWCSPH. Some conclusions are summed up at the end of this paper.

\section{SPH METHODOLOGY}

The basic formulation of SPH method consists of two steps of approximation: the kernel approximation and the particle approximation [21]. In the kernel approximation, the function and its derivatives are represented as integrations over the support domain. The particle approximation divides the support domain into discrete particles with own masses, densities, velocities, pressures and temperatures. For example, a function and its derivative can be written in the following forms: 


$$
\begin{aligned}
A(\mathbf{r}) & =\int A\left(\mathbf{r}^{\prime}\right) W\left(\mathbf{r}-\mathbf{r}^{\prime}, h\right) \mathrm{d} \mathbf{r}^{\prime} \\
& \approx \sum_{b} \frac{m_{b} A\left(\mathbf{r}_{b}\right)}{\rho_{b}} W\left(\mathbf{r}-\mathbf{r}_{b}, h\right) \\
\vec{\nabla} A(\mathbf{r}) & =\int_{A\left(\mathbf{r}^{\prime}\right) \vec{\nabla} W\left(\mathbf{r}-\mathbf{r}^{\prime}, h\right) \mathbf{d} \mathbf{r}^{\prime}} \\
& \approx \sum_{b} \frac{m_{b} A\left(\mathbf{r}_{b}\right)}{\rho_{b}} \vec{\nabla} W\left(\mathbf{r}-\mathbf{r}_{b}, h\right)
\end{aligned}
$$

where the subscript $b$ denotes the neighboring particles in the support domain, $W$ is the kernel function defined over the support domain, $\mathbf{r}$ is the position vector of the particle, $h$ is the smooth length, $m_{b}$ and $\rho_{b}$ denote the mass and the density of the particle in support domain, respectively.

To eliminate or alleviate possible tensile instability in SPH method when modeling fluid flows, a hyperbolic shaped kernel function is applied [22]:

$$
W(r, h)=\frac{1}{3 \pi h^{2}} \begin{cases}q^{3}-6 q+6, & 0 \leqslant q<1 \\ (2-q)^{3}, & 1 \leqslant q<2 \\ 0, & 2 \leqslant q\end{cases}
$$

where $q=\left|\mathbf{r}-\mathbf{r}_{b}\right| / h$.

\section{GOVERNING EQUATIONS AND SPH DISCRETIZATION}

\subsection{Governing equations}

In this section, the governing equations for incompressible SPH and weakly compressible SPH are discussed. Both of the two numerical schemes satisfy the following Lagrangian Navier-Stokes equations:

$$
\begin{gathered}
\frac{d \rho}{\mathrm{d} t}=-\rho \vec{\nabla} \cdot \mathbf{u} \\
\frac{\mathrm{d} \mathbf{u}}{\mathrm{d} t}=-\frac{1}{\rho} \vec{\nabla} P+v_{0} \nabla^{2} \mathbf{u}+\mathbf{F}
\end{gathered}
$$

where $\rho, \mathbf{u}, P, v_{0}$ and $\mathbf{F}$ denote the density, the velocity, the pressure, the laminar viscosity $\left(10^{-6} \mathrm{~m}^{2} / \mathrm{s}\right.$ for water in this paper) and the external body force exerted on water, respectively.

\subsection{SPH discretization}

On the basis of the SPH methodology presented in Section 2, the velocity gradient and pressure gradient can be expressed in SPH form as

$$
\begin{gathered}
(\rho \vec{\nabla} \cdot \mathbf{u})_{a}=-\sum_{b} m_{b} \mathbf{u}_{a b} \cdot \vec{\nabla}_{a} W\left(\mathbf{r}_{a}-\mathbf{r}_{b}, h\right) \\
\left(\frac{1}{\rho} \vec{\nabla} P\right)_{a}=\sum_{b} m_{b}\left(\frac{P_{a}}{\rho_{a}^{2}}+\frac{P_{b}}{\rho_{b}^{2}}\right) \cdot \vec{\nabla}_{a} W\left(\mathbf{r}_{a}-\mathbf{r}_{b}, h\right)
\end{gathered}
$$

where the subscripts $a$ and $b$ denote the particle under consideration and the neighboring particle in the support domain of the concerned particle, respectively, and $\mathbf{u}_{a b}=\mathbf{u}_{a}-\mathbf{u}_{b}$. 
Specifically, with Lo and Shao's suggestion [23], the Laplacian operator in the governing equation is expressed as

$$
\left(v_{0} \nabla^{2} \mathbf{u}\right)_{a}=\sum_{b} \frac{4 m_{b}\left(\mu_{a}+\mu_{b}\right) \mathbf{r}_{a b} \cdot \vec{\nabla} W_{a b}}{\left(\rho_{a}+\rho_{b}\right)^{2}\left(\left|\mathbf{r}_{a b}\right|^{2}+\eta^{2}\right)} \mathbf{u}_{a b}
$$

where $\mu_{a}=\rho_{a} v_{0}, \mu_{b}=\rho_{b} v_{0}, \mathbf{r}_{a b}=\mathbf{r}_{a}-\mathbf{r}_{b}, \eta$ is used to ensure a nonzero denominator and $\eta=0.1 h$.

\section{INCOMPRESSIBLE SPH ALGORITHM}

\subsection{SPH projection method}

The pressure projection method is applied here to ensure incompressibility. To reduce the occurrence of instability, a half-time step scheme is taken in this paper [24]. In this scheme, the time integration is accomplished through two loops in one time step, ensuring second order accuracy.

In the first loop, the position and the velocity of a fluid particle are firstly predicted as

$$
\begin{gathered}
\mathbf{r}^{*}=\mathbf{r}^{n}+\Delta t\left(\frac{3}{2} \mathbf{u}^{n}-\frac{1}{2} \mathbf{u}^{n-1}\right) \\
\mathbf{u}^{*}=\frac{4}{3} \mathbf{u}^{n}-\frac{1}{3} \mathbf{u}^{n-1}+\frac{2}{3} \Delta t\left(\mathbf{F}^{n+1}+v_{0} \nabla^{2} \mathbf{u}^{n}\right)
\end{gathered}
$$

where superscript $*$ denotes the estimated value and $n$ means the number of time step.

Then, a correction step is applied on the basis of the pressure force. Because the incompressibility ensures that the density does not change, the pressure of fluid particles and boundary particles are obtained by solving the PPE:

$$
\vec{\nabla} \cdot\left(\frac{\vec{\nabla} P^{n+1}}{\rho}\right)=\frac{3}{2 \Delta t} \vec{\nabla} \cdot \mathbf{u}^{*}
$$

where the left-hand side can be expressed in SPH form as:

$$
\left(\nabla \cdot \frac{1}{\rho} \nabla P\right)_{a} \approx \sum_{b} m_{b} \frac{8}{\left(\rho_{a}+\rho_{b}\right)^{2}} \frac{\left(P_{a}-P_{b}\right) \mathbf{r}_{a b} \cdot \vec{\nabla} W_{a b}}{\left(\left|\mathbf{r}_{a b}\right|^{2}+0.01 h^{2}\right)}
$$

The corrected velocity is expressed as

$$
\mathbf{u}^{n+1}=\mathbf{u}^{*}-\frac{2}{3} \frac{\Delta t}{\rho} \vec{\nabla} P^{n+1}
$$

The velocity and the position in half time step are obtained by

$$
\begin{gathered}
\mathbf{u}^{n+1 / 2}=\frac{1}{8}\left(3 \mathbf{u}^{n+1}+6 \mathbf{u}^{n}-\mathbf{u}^{n-1}\right) \\
\mathbf{r}^{n+1 / 2}=\mathbf{r}^{n}+\frac{\Delta t}{24}\left(2 \mathbf{u}^{n+1}+11 \mathbf{u}^{n}-\mathbf{u}^{n-1}\right)
\end{gathered}
$$

The second loop in this numerical scheme advances another half time step to obtain the velocity and position at time step $n+1$. 


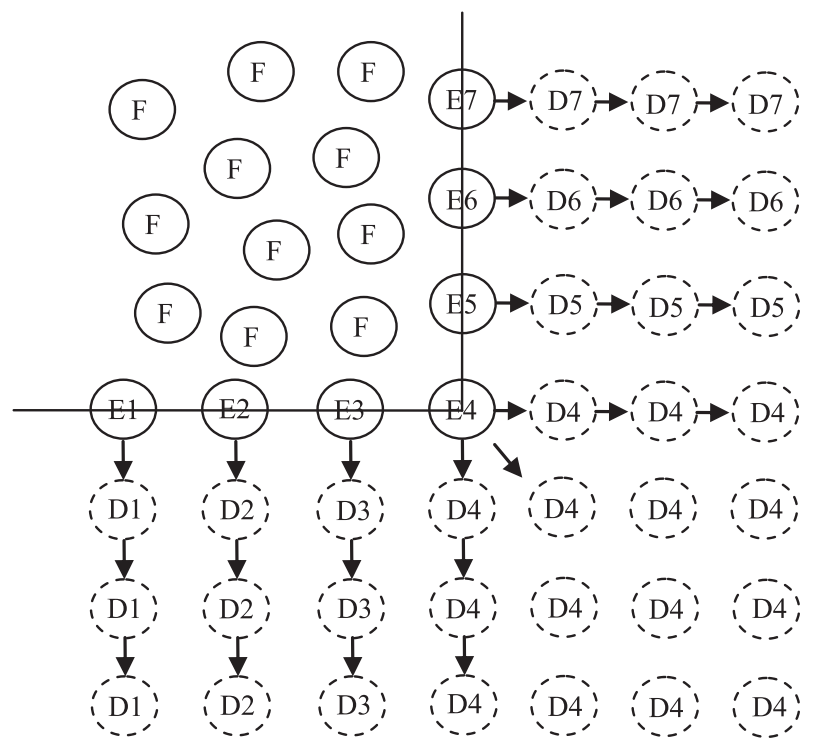

Figure 1. Illustration of boundary treatment in ISPH.

\subsection{Treatment of solid walls}

In ISPH, a common SBT is the combinational use of edge particles and dummy particles. On the basis of the work in literature [19], one layer of edge particles (named here as $E^{*}$ ) and three layers of dummy particles (named here as $D^{*}$ ) are placed on solid boundaries. The edge particles are involved in the solving of Poisson pressure equation. Dummy particles are used here to avoid the truncation of support domain for fluid particles near solid walls. The pressures of these dummy particles are given by the following formula to meet the Neumann boundary condition:

$$
P_{i}=P_{j}+\rho \mathbf{g} \cdot\left(\mathbf{r}_{i}-\mathbf{r}_{j}\right)
$$

where $i$ and $j$ denote to the dummy particle and its reference edge particle, respectively, $\mathbf{g}$ is the acceleration of gravity. The nearest edge particle is chosen as the reference particle for a dummy particle, as shown in Figure 1.

\subsection{Free surface tracking and treatment}

In ISPH, the widely used divergence of the position vectors is applied in the identification of free surface particles:

$$
\vec{\nabla} \cdot \mathbf{r}_{a}=\sum_{b} \frac{m_{b}}{\rho_{b}} \mathbf{r}_{a b} \cdot \vec{\nabla}_{a} W\left(\mathbf{r}_{a}-\mathbf{r}_{b}, h\right)
$$

For particles far from free surfaces, this divergence has a theoretical value of 2 in 2D. However, because of the loss of particles in the support domains of free surface particles, their divergence values of the position vectors shall be much less than 2 . In this paper, a threshold value of 1.5 is used to determine the free surface particles.

There are different ways to treat the detected free surface particles. One is to apply accurate Dirichlet boundary condition on the free surface particles, which means the pressures of all free surface particles are strictly set to zero. However, this treatment may lead to particle aggregation on free surfaces because of the lack of repulsive forces between free surface particles. Arne Bøckmann et al. [24] used another method to keep small pressure divergence between free surface particles. 
Similar to their works, in this paper, if identified as a free surface particle, Equation (12) is replaced by

$$
\left(\nabla \cdot \frac{1}{\rho} \nabla P\right)_{a}=\sum_{b} m_{b} \frac{8}{\left(\rho_{a}+\rho_{b}\right)^{2}} \frac{\left(2 P_{a}-P_{b}\right) \mathbf{r}_{a b} \cdot \vec{\nabla} W_{a b}}{\left(\left|\mathbf{r}_{a b}\right|^{2}+0.01 h^{2}\right)}
$$

\section{AN IMPROVED WEAKLY COMPRESSIBLE SPH MODEL}

\subsection{Formulation}

The WCSPH methods explicitly solve the Lagrangian Navier-Stokes equations. According to the SPH discretization scheme discussed in Section 3.2, the continuity and momentum equations can be expressed as

$$
\begin{gathered}
\frac{\mathrm{d} \rho_{a}}{\mathrm{~d} t}=-\sum_{b} m_{b}\left(\mathbf{u}_{a}-\mathbf{u}_{b}\right) \cdot \nabla_{a} W\left(\mathbf{r}_{a}-\mathbf{r}_{b}, h\right) \\
\frac{\mathrm{d} \mathbf{u}_{a}}{\mathrm{~d} t}=-\sum_{b} m_{b}\left(\frac{P_{a}}{\rho_{a}^{2}}+\frac{P_{b}}{\rho_{b}^{2}}\right) \cdot \vec{\nabla}{ }_{a} W\left(\mathbf{r}_{a}-\mathbf{r}_{b}, h\right)+\sum_{b} \frac{4 m_{b}\left(\mu_{a}+\mu_{b}\right) \mathbf{r}_{a b} \cdot \vec{\nabla} W_{a b}}{\left(\rho_{a}+\rho_{b}\right)^{2}\left(\left|\mathbf{r}_{a b}\right|^{2}+\eta^{2}\right)} \mathbf{u}_{a b}+\mathbf{g},
\end{gathered}
$$

The pressure value is obtained from an artificial equation of state, such as

$$
P=c^{2}\left(\rho-\rho_{0}\right)
$$

where $c$ and $\rho_{0}$ denote the numerical sound speed and the reference density $\left(1000 \mathrm{~kg} / \mathrm{m}^{3}\right.$, for water in this work), respectively.

Technically, the density variation should be kept within $1 \%$ to meet the assumption of weak compressibility. Hence, the numerical sound speed $c$ is usually chosen as at least 10 times bigger than the estimated maximum fluid velocity $[21,25]$.

\subsection{Density re-initialization}

In SPH, particles are not restricted in grids, making it more convenient to simulate a phenomenon with large deformations and strong discontinuities. However, the movement of fluid particles can lead to unstable number of neighboring particles within the support domain, causing false density variations and pressure oscillations in numerical calculation. A promising way to solve this problem is to re-initialize densities of all particles every a number of time steps. In this IWCSPH model, the MLS density re-initialization algorithm (Equations (22), (23), (24) and (25)) developed by Dilts $[11,12]$ is applied every 20 time steps.

$$
\begin{gathered}
\rho_{a}^{\mathrm{new}}=\sum_{b} \rho_{b} W_{a b}^{\mathrm{MLS}} \frac{m_{b}}{\rho_{b}}=\sum_{b} m_{b} W_{a b}^{\mathrm{MLS}} \\
W_{a b}^{\mathrm{MLS}}=\left[\beta_{0}+\beta_{x}\left(x_{a}-x_{b}\right)+\beta_{z}\left(z_{a}-z_{b}\right)\right] W_{a b} \\
{\left[\begin{array}{c}
\beta_{0} \\
\beta_{x} \\
\beta_{z}
\end{array}\right]=\left(\sum_{b} W_{a b} \mathbf{A} \frac{m_{b}}{\rho_{b}}\right)^{-1}\left[\begin{array}{c}
1 \\
0 \\
0
\end{array}\right]} \\
\mathbf{A}=\left[\begin{array}{ccc}
1 & x_{a}-x_{b} \\
x_{a}-x_{b} & \left(x_{a}-x_{b}\right)^{2} & \left(x_{a}-x_{b}\right)\left(z_{a}-z_{b}\right) \\
z_{a}-z_{b} & \left(x_{a}-x_{b}\right)\left(z_{a}-z_{b}\right) & \left(z_{a}-z_{b}\right)^{2}
\end{array}\right]
\end{gathered}
$$




\subsection{Improved coupled dynamic solid boundary treatment algorithm}

It is a great challenge for traditional WCSPH to find a proper and efficient way to implement solid boundary condition. Usually, two most widely used SBTs are the dynamic boundary condition and the repulsive boundary condition. However, the dynamic boundary condition cannot completely prevent the penetration of fluid particles, whereas the repulsive boundary condition causes the truncation in the support domains of fluid particles near solid boundaries and leads to pressure oscillations. The coupled dynamic SBT algorithm, developed by Shao et al. [26], combines both the dynamic boundary condition and repulsive boundary condition and is an effective implementation of the solid boundary condition.

In this improved WCSPH model, an improved coupled dynamic SBT algorithm is implemented in the SBT. This improved algorithm shows higher efficiency and gives more accurate results in numerical simulation.

Similar to the coupled dynamic SBT algorithm originally presented in literature [26], fluid particles approaching the repulsive boundary particles will experience the repulsive forces:

$$
\begin{gathered}
\mathbf{F}_{i j}=0.01 \bar{c}_{i j}^{2} \cdot \chi \cdot f(\eta) \cdot \frac{\mathbf{r}_{i j}}{\left|\mathbf{r}_{i j}\right|^{2}} \cdot m_{\text {fluid }} \\
\chi= \begin{cases}1-\frac{\left|\mathbf{r}_{i j}\right|}{h}, & 0<\left|\mathbf{r}_{i j}\right|<h \\
0, & \text { otherwise }\end{cases} \\
\eta=\frac{\left|\mathbf{r}_{i j}\right|}{0.5 h} \\
f(\eta)=\left\{\begin{array}{cc}
2 / 3, & 0<\eta<2 / 3 \\
\left(2 \eta-1.5 \eta^{2}\right), & 2 / 3<\eta<1 \\
0.5(2-\eta)^{2}, & 1<\eta<2 \\
0, & \text { otherwise }
\end{array}\right.
\end{gathered}
$$

where the subscript $i$ and $j$ denote the particle $i$ and the particle $j$, respectively, $m_{\text {fluid }}$ is the mass of the approaching fluid particle and $\overline{c_{i j}}$ is the average numerical sound velocity of the interacting fluid particle and boundary particle.

However, different from the original coupled dynamic SBT algorithm, the density re-initialization of boundary particles in this improved algorithm not only takes the fluid particles into account but also considers the boundary particles in the support domain. This modification increases numerical stability because the information of the concerned particle contributes to the particle approximation of the particle itself. Moreover, it is not necessary to implement this density re-initialization every time step because the small changes in solid boundary does not make much difference to the whole system in WCSPH. In this paper, the density re-initialization of boundary particles is applied every 20 time steps.

\subsection{Free surface condition}

The particle number density is used as the identification criteria for free surface particles in this IWCSPH model, which can be expressed as

$$
\rho_{a}=\sum_{b} \frac{m_{b}}{\rho_{b}} W\left(\mathbf{r}_{a}-\mathbf{r}_{b}, h\right)
$$

Theoretically, the particle number density of the fluid particle far from the free surface should be 1.0. However, for a free surface particle, because of its truncated support domain and less neighboring particles, the particle number density is much less than 1.0. In this paper, the particle with a particle number density less than 0.9 is identified as the free surface particle, and the pressure of the identified free surface particle is set to zero. 


\section{COMPARISON OF A WATER TANK WITH HYDROSTATIC PRESSURE}

The examination of hydrostatic pressure in a tank partially filled with still water is a benchmark problem, and it is quite a challenge for traditional SPH method to obtain a stable hydrostatic pressure [27]. In this section, the hydrostatic pressures obtained through the ISPH method and the improved WCSPH model are compared and discussed.

As shown in Figure 2, the breadth and height of the water tank are 1 and $1 \mathrm{~m}$, respectively. The depth of water column is $0.3 \mathrm{~m}$, and a pressure probe point (point $\mathrm{P}$ in Figure 2) is placed $0.1 \mathrm{~m}$ below the initial free surface on the left wall. Theoretically, the pressure value of this point should be a constant one of $\rho g d=981 \mathrm{~Pa}$. The total number of fluid particles used in this simulation is $N=100 \times 30$, with an initial particle spacing of $0.01 \mathrm{~m}$. The time step taken in the ISPH simulation is $10^{-4} \mathrm{~s}$, and in the IWCSPH simulation, the time step is $10^{-5} \mathrm{~s}$.

Figure 3 shows the pressure snapshots obtained by using ISPH and IWCSPH at $t=15 \mathrm{~s}$. This time instant is chosen to stay away from initial disturbance and examine the numerical stability of the two numerical models discussed above. For the convenience of comparisons, the calibration scale is kept uniform in the pressure snapshots obtained by ISPH and IWCSPH. It is observed that both models give smooth pressure fields, but the average pressure in the ISPH snapshot is higher than that in the IWCSPH snapshot.

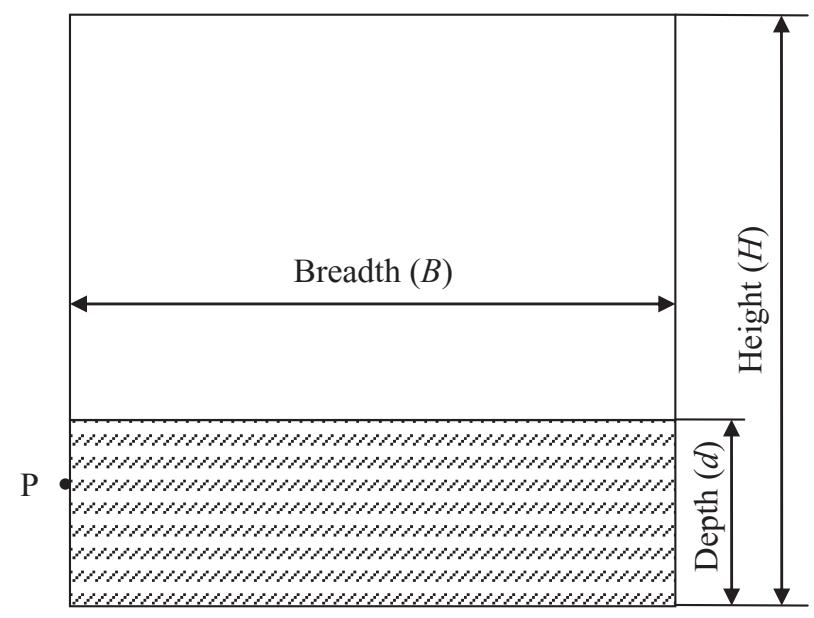

Figure 2. Illustration of the numerical water tank.

(a)

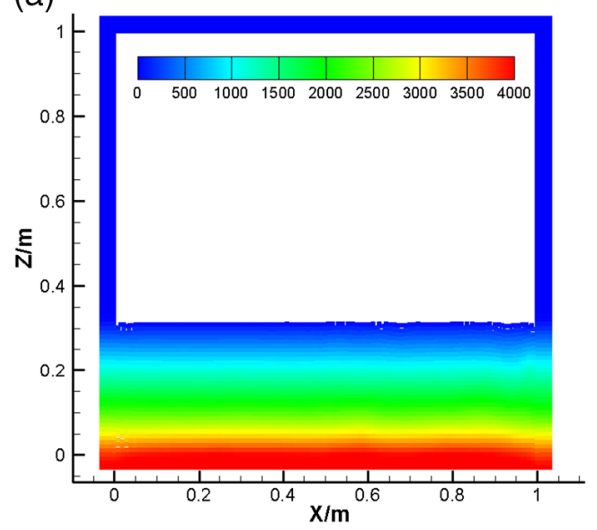

(b)

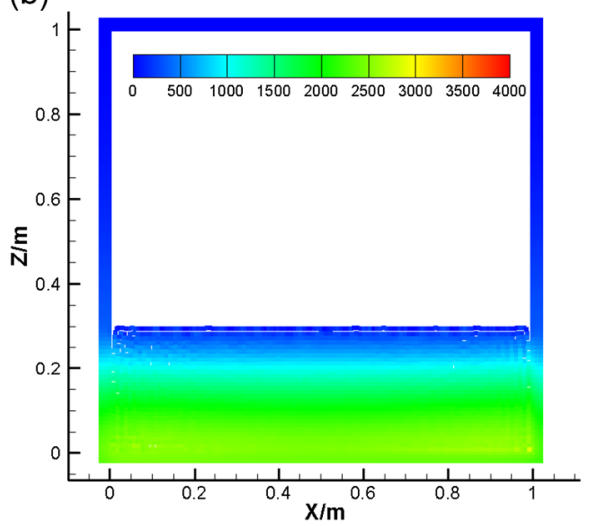

Figure 3. Pressure snapshots at $t=15 \mathrm{~s}$ : left, ISPH; right, improved WCSPH. 


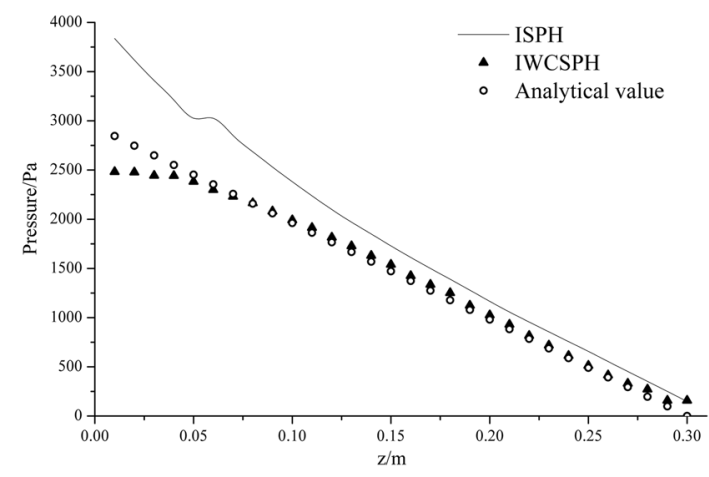

Figure 4. Pressure profiles along the middle section of the water tank.

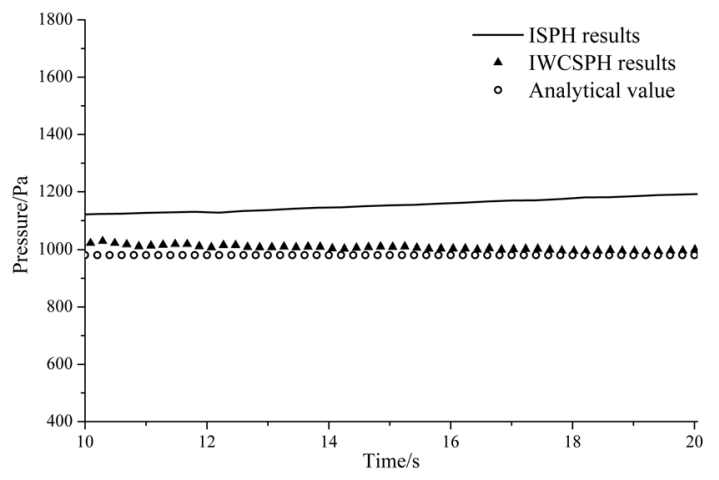

Figure 5. Pressure histories of the probe point $\mathrm{P}$.

Figure 4 demonstrates the comparison of pressure profiles along the middle section of the water tank $(x=0.5 \mathrm{~m})$ at $t=15 \mathrm{~s}$. It is observed that the pressure profile obtained by ISPH is significantly higher than the analytical solution, and the discrepancy becomes larger as the depth increases. The pressure profile obtained by IWCSPH is very close to the analytical pressure profile, except for some small discrepancies near the solid boundary area.

Figure 5 shows the pressure histories of the probe point P obtained by ISPH and IWCSPH. It is observed that both models give stable numerical results for this still water tank with hydrostatic pressure. However, the pressure history obtained by ISPH keeps rising as time marches, which may be due to ISPH's sensitivity to small disturbances. On the contrary, the pressure curve from IWCSPH is very close to the analytical solution and slightly keeps going down because of the small numerical diffusion in this model.

In general, both ISPH and IWCSPH give acceptable results for this hydrostatic test. The results of IWCSPH are about $10 \%$ lower than those of ISPH and are closer to the theoretical values. Hence, it can be concluded that IWCSPH is more accurate than ISPH in spatial and temporal pressure distributions for this benchmark problem with hydrostatic pressure.

\section{COMPARISON OF HYDRODYNAMIC EXAMPLES}

Although both ISPH and IWCSPH can simulate hydrostatic problems very well, hydrodynamic problems attract more interests in engineering applications. In this section, two typical problems with water impact and the periodical liquid sloshing are tested and discussed, respectively. Some conclusions are drawn on the basis of these analyses. 


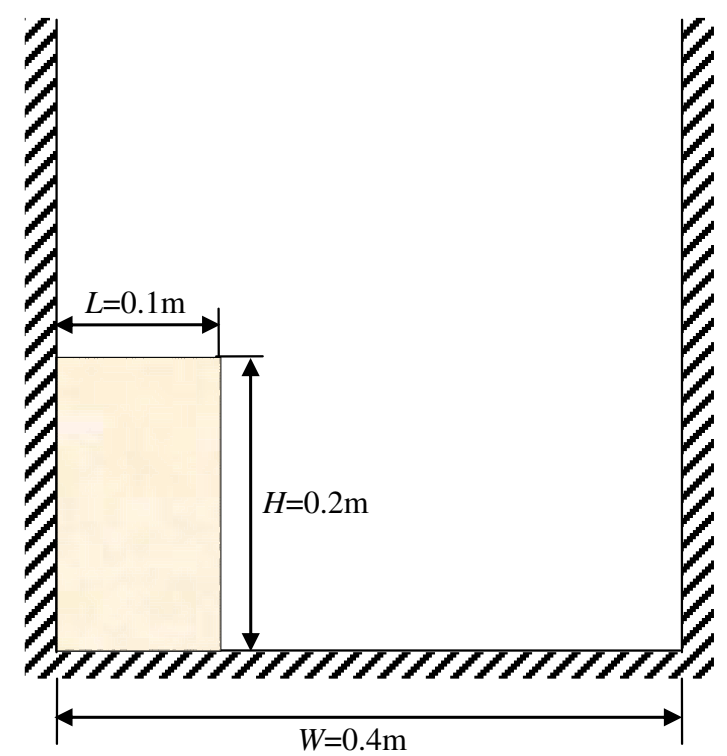

Figure 6. Initial setup of the first case of dam breaking.

\subsection{Dam breaking problem with large impact pressure}

Dam breaking is a common and important benchmark problem for numerical simulations of free surface incompressible flows [28]. It usually comes with lots of complex phenomenon, including changing and breakup of free surfaces, splashing and strong impact against solid boundaries. In this section, two cases of 2D dam breaking are presented and discussed to comparatively investigate the reliability of the ISPH method and the IWCSPH model. Meanwhile, some comparisons and analyses of the computational costs are offered in this section.

In the first case of dam breaking, the experimental domain is $W=0.4 \mathrm{~m}$ long, and the initial dimension of water column is $L \times H=0.1 \mathrm{~m} \times 0.2 \mathrm{~m}$, as shown in Figure 6 . The total number of fluid particles is $N=40 \times 80$, with the initial particle spacing of $d_{0}=0.0025 \mathrm{~m}$. In ISPH simulation, the time step is taken as $10^{-4} \mathrm{~s}$, and in IWCSPH simulation it is $10^{-5} \mathrm{~s}$.

The numerical results at two different moments are presented in Figure 7. The results obtained by ISPH and IWCSPH are compared at dimensionless time moments of $t(g / H)^{0.5}=1.96$ and 4.55 , respectively. It is observed that both methods can obtain reasonable flow pattern and smooth pressure fields for areas far from the solid boundaries and free surfaces. IWCSPH can obtain better details in the flow jet and cavity. Most importantly, ISPH is associated with bigger oscillation near the solid boundaries and free surfaces, and small disturbance can lead to numerical instability. This is due to the different schemes in calculating pressure in ISPH and WCSPH. In ISPH, pressure is obtained through solving a system matrix equation (describing PPE) on the entire computational domain; small perturbations in boundary or free surface areas can simultaneously propagate across the entire computational domain and can further lead to bigger oscillations and instability in the numerical simulation. In contrast, in WCSPH, the pressure is obtained from the equation of state and is related to the approximation of density, which is conducted only within the support domain of a concerned particle. Numerical oscillations or small errors at a concerned particle only influence neighboring particles within the support domain rather than the entire computational domain at the same step. Their influences to particles far away from the concerned particle are effective only at later time steps. In other words, in ISPH, the influence of numerical oscillation is more direct and global. Any numerical disturbance can influence the entire computational domain at the same time step. However, in WCSPH, the influence of numerical oscillation is indirect and local. The propagation of possible numerical disturbance can be smeared and vanished over a number of time steps. Hence, ISPH is more sensitive to numerical oscillations and easier to induce numerical instability, whereas WCSPH is more flexible. 
(a)

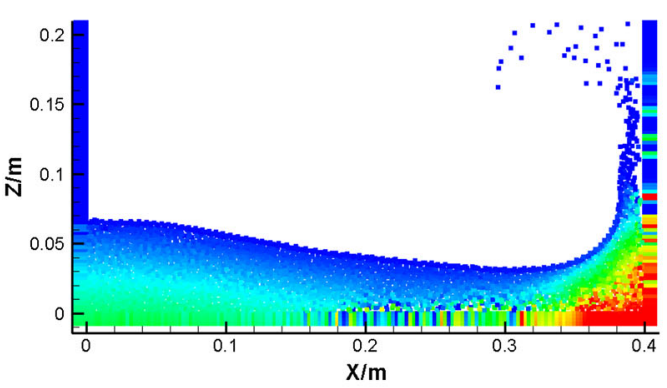

(c)
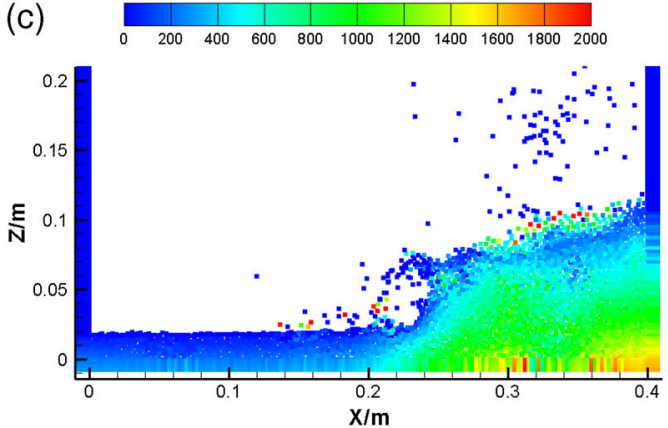

(b)
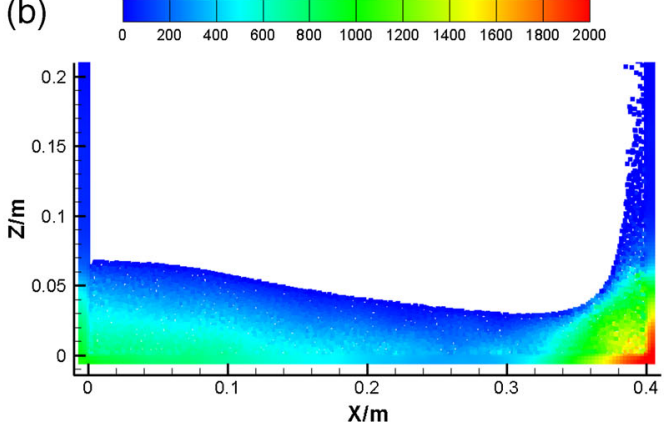

(d)

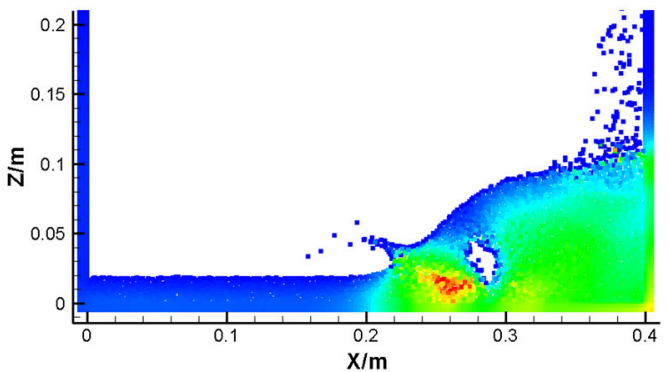

Figure 7. Comparison (pressure fields) of the first case of dam breaking: (a) ISPH at $t(g / H)^{0.5}=1.96$; (b) IWCSPH at $t(g / H)^{0.5}=1.96$; (c) ISPH at $t(g / H)^{0.5}=4.55$; (d) IWCSPH at $t(g / H)^{0.5}=4.55$.

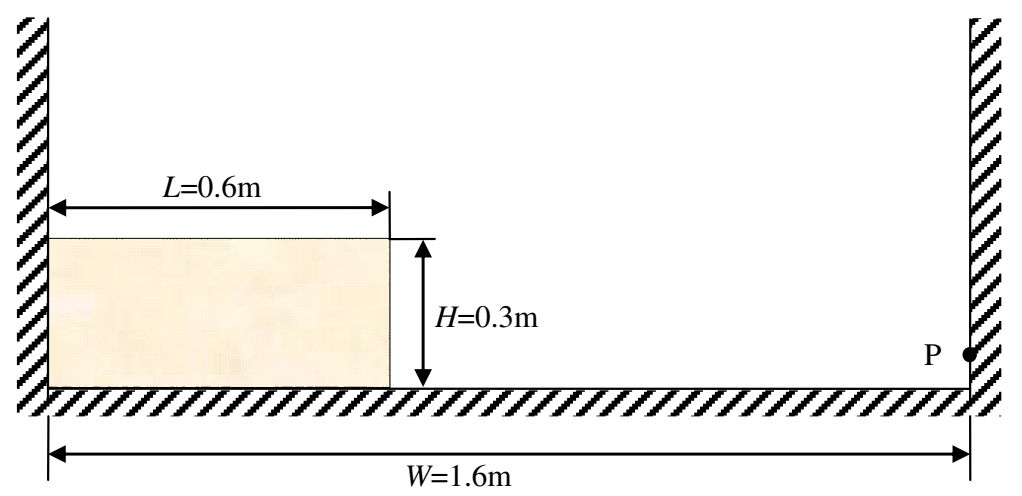

Figure 8. Initial setup of the second case of dam breaking.

Here, we consider another case of dam breaking and offer some quantitative comparison between the results obtained by ISPH and IWCSPH. In this example of dam breaking, the experimental domain is $W=1.6 \mathrm{~m}$ long, and the initial dimension of water column is $L \times H=0.6 \mathrm{~m} \times 0.3 \mathrm{~m}$, as shown in Figure 8. The total number of fluid particles is $N=300 \times 150$, with the initial particle spacing of $d_{0}=0.002 \mathrm{~m}$. In ISPH simulation, the time step is taken as $5 \times 10^{-5} \mathrm{~s}$, and in IWC$\mathrm{SPH}$ simulation, it is $5 \times 10^{-6} \mathrm{~s}$. To track and analyze the time variations of pressure, a pressure measurement point $\mathrm{P}$ is located on the right wall and $0.06 \mathrm{~m}$ above the bottom.

The numerical results at two special instants are presented in Figure 9. The results obtained by ISPH and IWCSPH are compared at dimensionless time instants of $t(g / H)^{0.5}=3.2$ and 6.4, respectively. In this example, both ISPH and IWCSPH give generally smooth pressure fields and present those important details such as flow cavity and flow jet. However, it is observed that in the results obtained by ISPH, the pressure field inside the flow still suffers from some incorrect oscillations and discontinuities, especially in places where strong impacts happen. Meanwhile, the water splash 
(a)
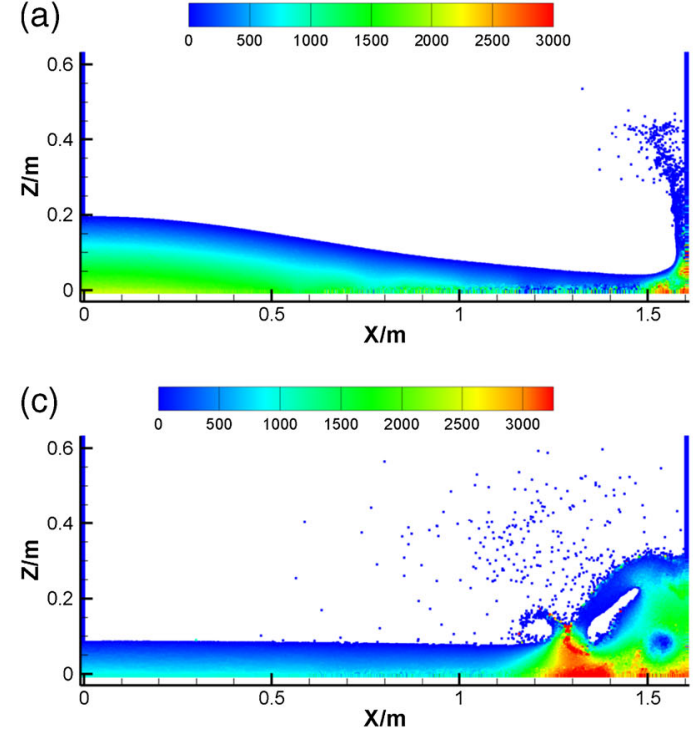

(b)

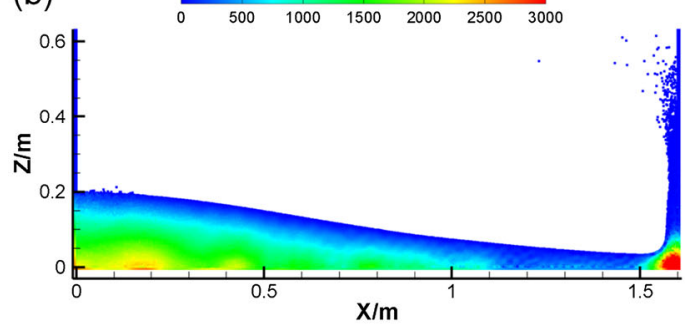

(d)

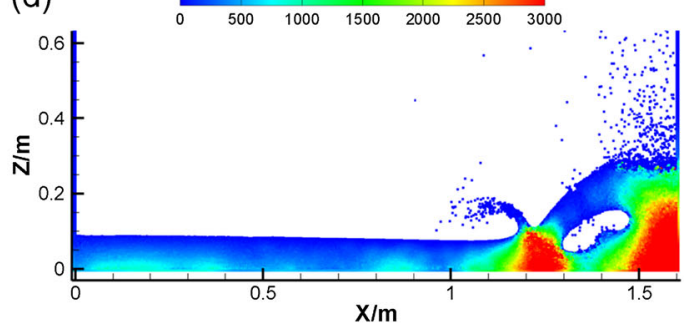

Figure 9. Comparison (pressure fields) of the second case of dam breaking: (a) ISPH at $t(g / H)^{0.5}=3.2$; (b) IWCSPH at $t(g / H)^{0.5}=3.2$; (c) ISPH at $t(g / H)^{0.5}=6.4$; (d) IWCSPH at $t(g / H)^{0.5}=6.4$.

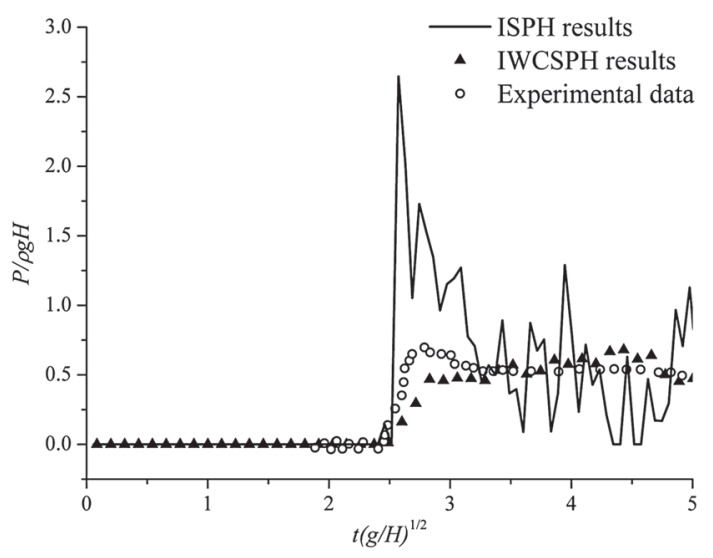

Figure 10. Comparison of time variations of dimensionless pressure on point $\mathrm{P}$.

in ISPH simulation is much more severe than that in IWCSPH simulation, which indicates that the IWCSPH is more flexible and stable than ISPH.

A detailed comparison of the pressure history on point $\mathrm{P}$ is shown in Figure 10. The pressure curves obtained by ISPH and IWCSPH are compared with experimental data [29]. It is observed that the pressure curve obtained by ISPH simulation oscillates more greatly than that obtained by IWCSPH simulation, and the oscillation amplitude is so big that it has concealed the valuable information about the pressure history on point P. Specifically, when strong impact happens, the instability of ISPH becomes much more severe, which is shown as the exorbitant pressure during dimensionless time from 2.6 to 3.0 in Figure 10. In contrast, the pressure history obtained in IWCSPH simulation is generally smooth. During the occurrence of strong impact, the IWCSPH captured the impact pressure very well. Although the pressure value is smaller than the experimental data, the discrepancy is acceptable for engineering applications.

To sum up, for the two dam breaking cases, both ISPH and IWCSPH can obtain reasonable flow patterns with smooth pressure fields in bulky flow region. However, IWCSPH is more accurate, more flexible and can capture more flow details such as jets and cavity. ISPH is more sensitive to numerical oscillations, and it is easier to cause instability. 
Table I. Comparison of computational time.

\begin{tabular}{lcccc}
\hline & Number of fluid particles & Physical time & CPU time (ISPH) & CPU time (IWCSPH) \\
\hline Dam breaking 1 & 3000 & $1.0 \mathrm{~s}$ & $5159 \mathrm{~s}$ & $5449 \mathrm{~s}$ \\
Dam breaking 2 & 45,000 & $1.5 \mathrm{~s}$ & $54.5 \mathrm{~h}$ & $40.8 \mathrm{~h}$ \\
\hline
\end{tabular}

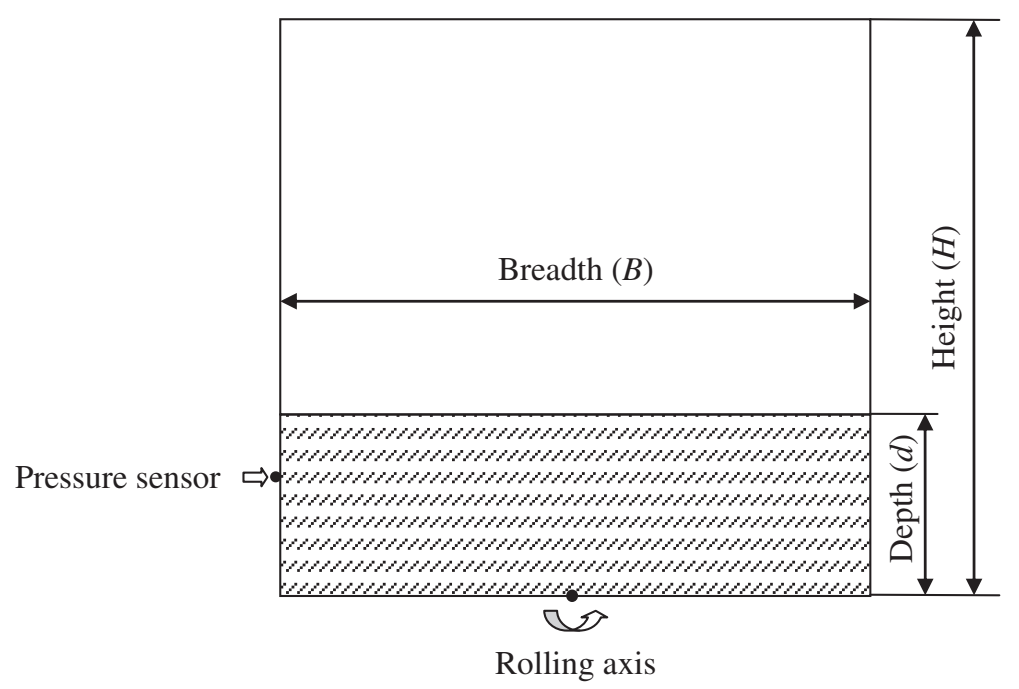

Figure 11. Illustration of the sloshing system.

Computational cost can be a major concern for both ISPH and WCSPH. ISPH is a semi-implicit method that can use big time step but is time-consuming in solving the PPE at every time step. WCSPH is an explicit method, which uses comparatively smaller time step. Here, we give a detailed comparison of the computational cost for the presented ISPH and IWCSPH models (in Table I). It is noted that as ISPH needs to solve PPE at every time step, the sparse matrix solver can be very important. In this work, a parallel sparse matrix solver (PARDISO in Intel ${ }^{\circledR}$ Math Kernel Library) is used. All numerical simulations are conducted on a computer with a dual-core processor. It is found that although the time step in ISPH is generally 10 times bigger than that in IWCSPH, ISPH does not show significant advantages over IWCSPH in computational cost, and as the computational scale (i.e., the number of particles) increases, IWCSPH gradually shows higher efficiency.

\subsection{Liquid sloshing problem with periodically changing hydrodynamic pressure}

Sloshing refers to the periodic liquid movement in a partially filled container due to external excitations [30]. It is a common and important phenomenon in engineering field. In this section, an example of liquid sloshing is presented to test the adaptabilities of ISPH and IWCSPH in periodic hydrodynamic problems.

As shown in Figure 11, the geometry of the water tank is $B \times H=1 \mathrm{~m} \times 1 \mathrm{~m}$, and the water depth is $d=0.3 \mathrm{~m}$. The total number of fluid particles is $N=100 \times 30$, with the initial particle spacing of $d_{0}=0.01 \mathrm{~m}$. A pressure sensor is located on the left wall and is $0.1 \mathrm{~m}$ below the initial free surface. The tank rolls around the axis at the center of its bottom, and the rolling angle $\alpha$ follows the motion law expressed as

$$
\alpha=A \sin (\omega t)
$$

where $A, \omega$ and $t$ denote the rolling amplitude, the excitation circular frequency and time, respectively. In this example, $A=5^{\circ}, \omega=0.95 \mathrm{rad} / \mathrm{s}$.

Figures 12 and 13 show the pressure fields obtained by using ISPH and IWCSPH at different time instants. It is observed that both of the two models give smooth pressure pictures, but the 
(a)

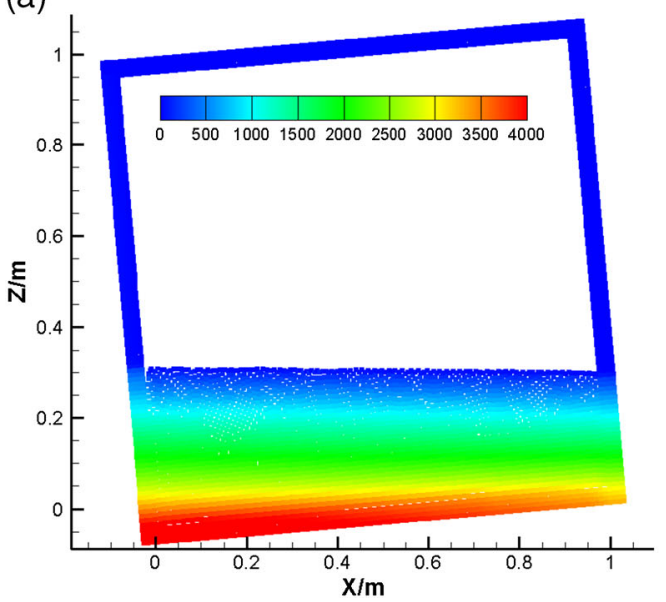

(b)

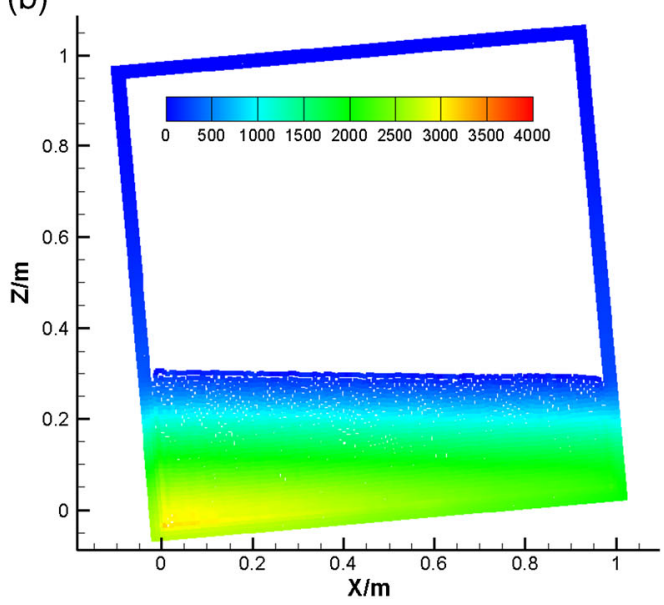

Figure 12. Comparison of the pressure fields, $t=1.25 \mathrm{~T}$ : left, ISPH; right, IWCSPH.

(a)

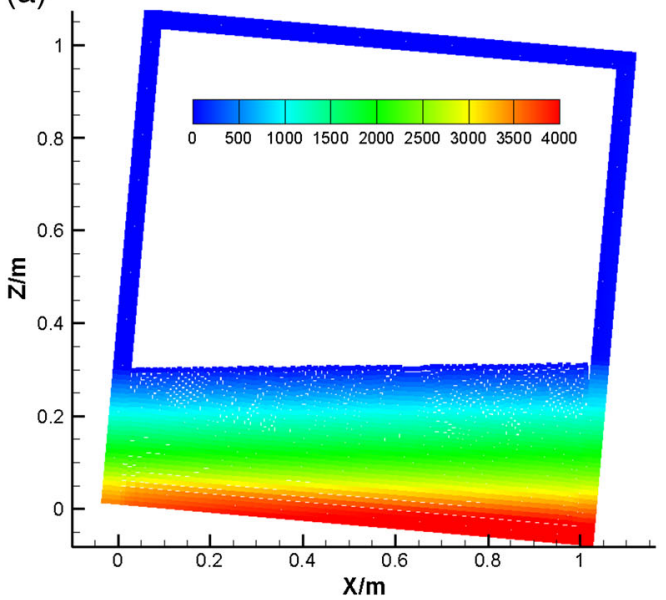

(b)

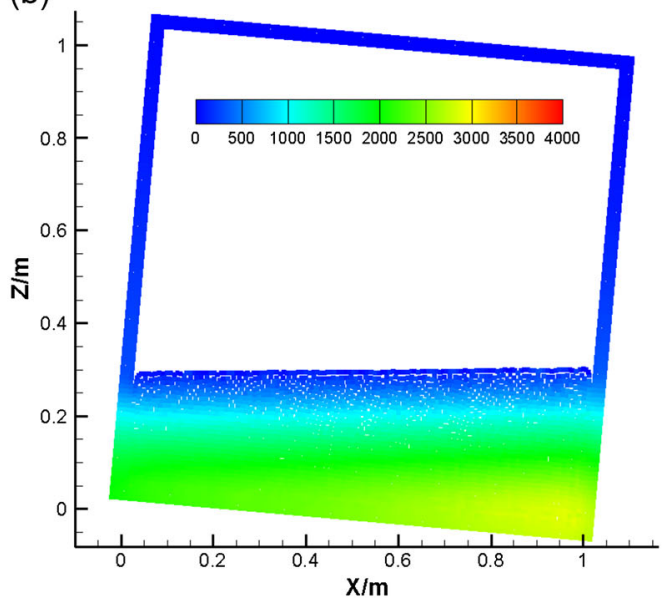

Figure 13. Comparison of the pressure fields, $t=1.75 \mathrm{~T}$ : left, ISPH; right, IWCSPH.

pressure obtained by ISPH is a little bit higher than that obtained by IWCSPH. To find out which result is more reliable, the pressure history on the pressure sensor point is recorded and presented in Figure 14. Comparing the curves obtained in numerical simulations with the experimental data [31], it is found that although the average pressure value decreases a little as the time marches, the pressure curve obtained by the IWCSPH model is in better agreement with the real condition. For the pressure curve obtained by ISPH, although a similar shape is retained, the average pressure keeps rising and deviates from the experimental data more greatly compared with that obtained by IWCSPH. The greater discrepancies between ISPH results and the experimental data may still come from the instability of ISPH algorithm.

To eliminate the possible worry of grid dependence, more particles are used for a comparative study. The particle spacing in this test is $d_{0}=0.005 \mathrm{~m}$. The pressure curves obtained by ISPH simulation and IWCSPH simulation are presented in Figure 15. It is observed that, compared with the previous sloshing test, there are no obvious differences in IWCSPH simulation with more particles. Although the results obtained by ISPH simulation with more particles show slightly better agreement with the experimental data compared with the previous ISPH results with fewer particles, they do not have significant advantages over IWCSPH results. Moreover, when the particle number increases, as stated in the previous section, IWCSPH shows higher efficiency than ISPH. 


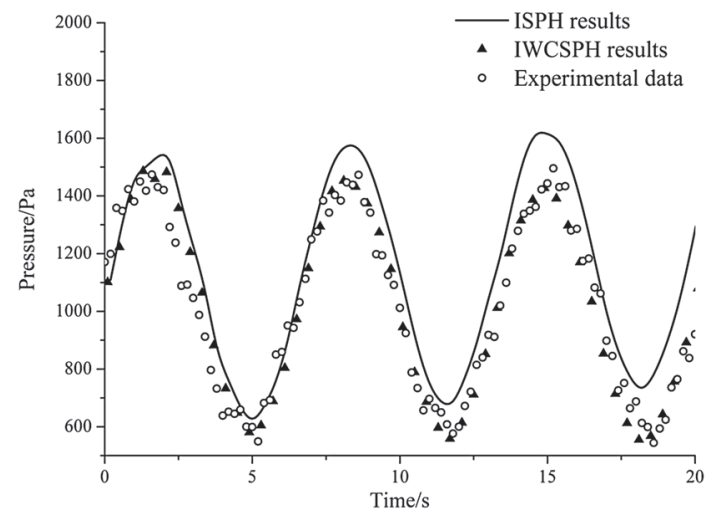

Figure 14. Comparison of the pressure histories on point $\mathrm{P}$.

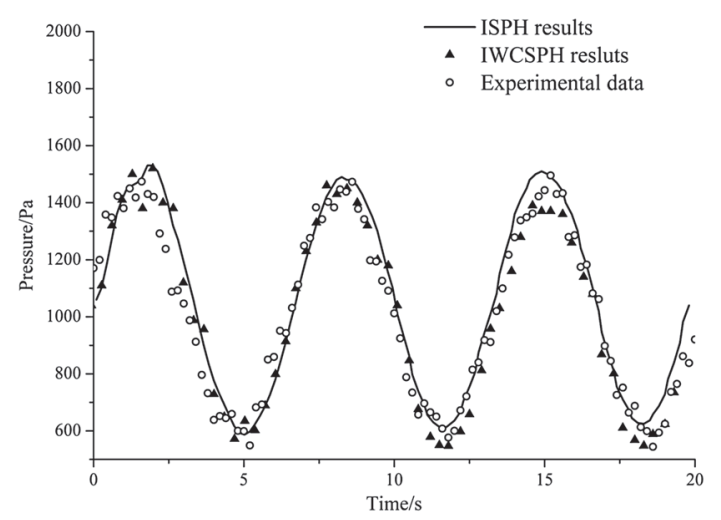

Figure 15. Pressure histories on point $\mathrm{P}$ obtained by numerical simulations with more particles.

Therefore, it can be concluded that for this liquid sloshing problem with periodically changing hydrodynamic pressure, the IWCSPH model also gives better results than ISPH.

\section{CONCLUSIONS AND REMARKS}

ISPH and WCSPH are two approaches in modeling incompressible flows within the frame of SPH. ISPH is a semi-implicit method that needs to solve the PPE, and WCSPH is an explicit method that is based on the weakly compressible assumption of the incompressible fluid. As ISPH and WCSPH are very popular, although reported results are usually inconsistent, in this paper, we present a comprehensive study of the ISPH method and an improved weakly compressible SPH method in free surface flows. Both methods solve the Navier-Stokes equations in Lagrangian form, and no artificial viscosity is used. The ISPH algorithm presented in this paper is based on the classical SPH projection method with some common treatments on solid boundaries and free surfaces. The IWCSPH model includes some advanced corrective algorithms in density approximation and SBT.

Three typical numerical examples, including a benchmark problem with hydrostatic pressure, a dam breaking problem with large impact pressure and a liquid sloshing problem with periodically changing hydrodynamic pressure, are comparatively analyzed with ISPH and IWCSPH. For these three representative examples, it is found that, in general, both ISPH and IWCSPH can well capture the flow patterns with smooth pressure fields. For the benchmark problem with hydrostatic pressure, IWCSPH is more accurate than ISPH in spatial and temporal pressure distributions. For dam breaking problem and a liquid sloshing problem, IWCSPH is more accurate and more stable, whereas ISPH is more sensitive to numerical oscillations and is easier to cause instability. And, 
therefore, the IWCSPH is more suitable for problems with violent water impact and fluid-structure interactions.

Another point is that although ISPH can use much bigger time steps, it needs to solve the timeconsuming sparse matrix equation. Therefore, ISPH is not superior to IWCSPH in computational cost. For problems with a large number of particles, IWCSPH can even be more efficient than ISPH. Moreover, as the IWCSPH code in this paper is a serial code and the ISPH code is partially parallelled, the IWCSPH may be more competitive after parallelization.

It is known that the conventional WCSPH usually suffers from low accuracy with high pressure oscillations. Although using numerical techniques such as higher order SPH approximation schemes and artificial viscosity can greatly improve WCSPH, in the presented IWCSPH, only a simple MLS approach for re-initializing density and an enhanced SBT algorithm are used. It is straightforward to implement on existing WCSPH without introducing inherent factors unsuitable for comparisons with ISPH.

In conclusion, although the ISPH method may have some advantages over traditional WCSPH method, the presented IWCSPH is more attractive than ISPH as it is more accurate and more stable with comparable or even less computational efforts.

\section{ACKNOWLEDGEMENTS}

The present work is supported by the National Natural Science Foundation of China $(11172306,51221961$ and 51279030), the National Key Basic Research Special Foundation of China (2010CB832704 and 2013CB036101) and the Scientific Project for High-tech Ships: Key Technical Research on the Semiplanning Hybrid Fore-body Trimaran.

\section{REFERENCES}

1. Liu GR, Liu MB. Smoothed Particle Hydrodynamics: A Meshfree Particle Method. World Scientific Pub Co Inc: Singapore, 2003.

2. Gingold RA, Monaghan JJ. Smooth Particle Hydrodynamics: theory and application to non spherical stars. Monthly Notices of the Royal Astronomical Society 1977; 181:375-389.

3. Liu MB, Liu GR. Smoothed particle hydrodynamics (SPH): an overview and recent developments. Archives of Computational Methods in Engineering 2010; 17:25-76.

4. Monaghan JJ. Smoothed particle hydrodynamics and its diverse applications. Annual Review of Fluid Mechanics 2012; 44:323-346.

5. Marrone S, Antuono M, Colagrossi A, Colicchio G, Touzé DL, Graziani G. $\delta$-SPH model for simulating violent impact flows. Computer Methods in Applied Mechanics and Engineering 2011; 200:1526-1542.

6. Delorme L, Colagrossi A, Souto Iglesias A, Rodriguez RZ, Vera EB. A set of canonical problems in sloshing, Part I: Pressure field in forced roll—comparison between experimental results and SPH. Ocean Engineering 2009; 36:168-178.

7. Chen JK, Beraun JE. A generalized smoothed particle hydrodynamics method for nonlinear dynamic problems. Computer Methods in Applied Mechanics and Engineering 2000; 190:225-239.

8. Liu MB, Liu GR. Restoring particle consistency in smoothed particle hydrodynamics. Applied Numerical Mathematics 2006; 56:19-36.

9. Liu MB, Xie WP, Liu GR. Modeling incompressible flows using a finite particle method. Applied Mathematical Modeling 2005; 29:1252-1270.

10. Colagrossi A, Landrini M. Numerical simulation of interfacial flows by smoothed particle hydrodynamics. Journal of Computational Physics 2003; 191:448-475.

11. Dilts GA. Moving-least-squares-particle hydrodynamics-i. consistency and stability. International Journal for Numerical Methods in Engineering 1999; 44:1115-1155.

12. Dilts GA. Moving-least-squares-particle hydrodynamics-ii. conservation and boundaries. International Journal for Numerical Methods in Engineering 2000; 48:1503-1524.

13. Liu MB, Shao JR, Chang JZ. On the treatment of solid boundary in smoothed particle hydrodynamics. SCIENCE CHINA Technological Sciences 2012; 55(1):244-254.

14. Zheng X, Duan WY, Ma QW. A new scheme for identifying free surface particles in improved SPH. Science China 2012; 55:1454-1493.

15. Shao SD, Ji CM, Graham DI, Reeve DE, James PW, Chadwick AJ. Simulation of wave overtopping by an incompressible SPH model. Coastal Engineering 2006; 53:723-735.

16. Cummins SJ, Rudman M. An SPH Projection Method. Journal of Computational Physics 1999; 152:584-607.

17. Rafiee A, Thiagarajan KP. An SPH projection method for simulating fluid-hypoelastic structure interaction. Computer Methods in Applied Mechanics and Engineering 2009; 198:2785-2795. 
18. Liu X, Xu HH, Shao SD, Lin PZ. An improved incompressible SPH model for simulation of wave-structure interaction. Computers \& Fluids 2013; 71:113-123.

19. Lee E-S, Moulinec C, Xu R, Violeau D, Laurence D, Stansby P. Comparisons of weakly compressible and truly incompressible algorithms for the SPH mesh free particle method. Journal of Computational Physics 2008; 227:8417-8436.

20. Shadloo MS, Zainali A, Yildiz M, Suleman A. A robust weakly compressible SPH method and its comparison with an incompressible SPH. International Journal for Numerical Methods in Engineering 2012; 89:939-956.

21. Monaghan JJ. Simulating Free-Surface Flows with SPH. Journal of Computational Physics 1994; 110:399-406.

22. Yang XF, Liu MB. Improvement on stress instability in smoothed particle hydrodynamics. Acta Physica Sinica 2012; 61:224701-1-224701-8.

23. Lo EYM, Shao SD. Simulation of near-shore solitary wave mechanics by an incompressible SPH method. Applied Ocean Research 2002; 24:275-286.

24. Bøckmann A, Shipliova O, Skeie G. Incompressible SPH for free surface flows. Computers \& Fluids 2012; 67:138-151

25. Liu MB, Liu GR, Zong Z. An Overview on Smoothed Particle Hydrodynamics. International Journal of Computational Methods 2008; 5:135-188.

26. Shao JR, Li HQ, Liu GR, Liu MB. An improved SPH method for modeling liquid sloshing dynamics. Computers \& Structures 2012; 100-101:18-26.

27. Oger G, Doring M, Alessandrini B, Ferrant P. An improved SPH method: Towards higher order convergence. Journal of Computational Physics 2007; 225:1472-1492.

28. Shao SD, Lo EYM. Incompressible SPH method for simulating Newtonian and non-Newtonian flows with a free surface. Advances in Water Resources 2003; 26:787-800.

29. Lee TH, Zhou ZQ, Cao YS. Numerical simulations of hydraulic jumps in water sloshing and water impacting. Journal of Fluids Engineering 2002; 124:215-226.

30. Faltinsen OM, Timokha AN. Sloshing. Cambridge University Press: Cambridge, UK, 2009.

31. Chen Z, Zong Z, Li HT, Li J. An investigation into the pressure on solid walls in 2D sloshing using SPH method. Ocean Engineering 2013; 59:129-141. 